\title{
Research Article \\ Hearing Phenotypes of Patients with Hearing Loss Homozygous for the GJB2 c.235delc Mutation
}

\author{
Chang Guo, ${ }^{1,2,3}$ Sha-Sha Huang, ${ }^{1,2,3}$ Yong-Yi Yuan $\mathbb{D}^{1,2,3}$ Ying Zhou, ${ }^{1,2,3}$ Ning Wang, 4 \\ Dong-Yang Kang, ${ }^{1,2,3}$ Su-Yan Yang, ${ }^{1,2,3}$ Xin Zhang, ${ }^{1,2,3}$ Xue Gao $\mathbb{D}^{1,2,3,5}$ and Pu Dai ${ }^{1,2,3}$ \\ ${ }^{1}$ College of Otolaryngology Head and Neck Surgery, Chinese PLA General Hospital, Chinese PLA Medical School, 28 Fuxing Road, \\ Beijing 100853, China \\ ${ }^{2}$ National Clinical Research Center for Otolaryngologic Diseases, State Key Lab of Hearing Science, Ministry of Education, China \\ ${ }^{3}$ Beijing Key Lab of Hearing Impairment Prevention and Treatment, Beijing, China \\ ${ }^{4}$ Department of Pediatric Surgery, Chinese PLA General Hospital, Chinese PLA Medical School, 28 Fuxing Road, \\ Beijing 100853, China \\ ${ }^{5}$ Department of Otolaryngology, PLA Rocket Force Characteristic Medical Center, 16\# XinWai Da Jie, Beijing 100088, China
}

Correspondence should be addressed to Xue Gao; mixueer0110@126.com and Pu Dai; daipu301@vip.sina.com

Received 12 April 2020; Revised 18 June 2020; Accepted 30 June 2020; Published 1 August 2020

Academic Editor: Renjie Chai

Copyright (C) 2020 Chang Guo et al. This is an open access article distributed under the Creative Commons Attribution License, which permits unrestricted use, distribution, and reproduction in any medium, provided the original work is properly cited.

\begin{abstract}
Hereditary hearing loss is characterized by remarkable phenotypic heterogeneity. Patients with the same pathogenic mutations may exhibit various hearing loss phenotypes. In the Chinese population, the c.235delC mutation is the most common pathogenic mutation of GJB2 and is closely related to hereditary recessive hearing loss. Here, we investigated the hearing phenotypes of patients with hearing loss associated with the homozygous c.235delC mutation, paying special attention to asymmetric interaural hearing loss. A total of 244 patients with the GJB2 c.235delC homozygous mutation encountered from 2007 to 2015 were enrolled. The severity of hearing loss was scaled with the American Speech-Language-Hearing Association (ASHA). Auditory phenotypes were analyzed, and three types of interaural asymmetry were defined based on audiograms: Type A (asymmetry of hearing loss severity), Type B (asymmetry of audiogram shape), and Type C (Type A plus Type B). Of the 488 ears (244 cases) examined, 71.93\% (351) presented with profound hearing loss, 14.34\% (70) with severe hearing loss, and $9.43 \%$ (46) with moderate to severe hearing loss. The most common audiogram shapes were descending $(31.15 \%)$ and flat $(24.18 \%)$. A total of $156(63.93 \%)$ of the 244 patients exhibited asymmetric interaural hearing loss in terms of severity and/or audiogram shape. Type A was evident in 14 of these cases, Type B in 106, and Type C in 36. In addition, 211 of 312 ears (67.63\%) in the interaural hearing asymmetry group showed profound hearing loss, and 59 (18.91\%) exhibited severe hearing loss, with the most common audiogram shapes being flat (27.88\%) and descending $(22.12 \%)$. By contrast, in the interaural hearing symmetry group, profound hearing loss was observed in 140 ears $(79.55 \%)$, and the most common audiograms were descending (46.59\%) and residual (21.59\%). Hearing loss associated with the GJB2 c.235delC homozygous mutation shows diverse phenotypes, and a considerable proportion of patients show bilateral hearing loss asymmetry.
\end{abstract}

\section{Introduction}

Hearing loss (HL) is one of the most common neurosensory impairments in humans [1]. The World Health Organization estimated in 2020 that over 5\% of the world's population (approximately 466 million people) suffer from HL [2].
Sensorineural HL (SNHL) is the most common form of HL and typically is caused by a loss of functional sensory hair cells (HCs) and supporting cells (SCs) within the cochlea $[3,4]$. HCs and SCs develop from common progenitor cells within the prosensory domain of the developing cochlea [5]. HCs transfer mechanical vibration into an acoustic 
electrical signal, which is then transmitted to the auditory cortex via spiral ganglion neurons (SGNs). SCs are important cells that support HCs and hold the potential to regenerate HCs once damaged [6-8]. HCs and SCs are very sensitive and vulnerable to stress and damage, classified mainly as genetic factors, environmental factors, ototoxic drugs, aging, inflammation, and other unknown etiologies [9-11]. Among these, genetic factors are responsible for approximately 50$60 \%$ of cases of HL [12]. Whereas $70 \%$ of genetic HL cases are nonsyndromic HL (NSHL), 30\% are syndromic. Approximately $80 \%$ of NSHL cases are inherited via an autosomal recessive mode, whereas other cases are inherited in an autosomal dominant, X-linked, or mitochondrial mode $[13,14]$. To date, more than 100 genes have been shown to cause NSHL [15]. Despite this, the most common cause of NSHL is mutations in GJB2 [16, 17].

The GJB2 gene encodes a $26 \mathrm{kDa}$ gap junction protein known as connexin $26(\mathrm{Cx} 26)$ [18]. Cx26 consists of an Nterminal helix, four transmembrane helices (TM1-4), two extracellular loops (E1 and E2), a cytoplasmic loop (CL), and a C-terminus [19]. Cx26 is expressed in the inner ear, which contains SCs, stria vascularis, spiral ligament, and spiral limbus [20]. Cx26 is associated with proteins that form a transmembrane hexameric gap junction channel known as a connexon. These channels are believed to play a role in the recycling of potassium ions from HCs to the endolymph $[21,22]$. GJB2 is involved in a series of physiological hearing processes including cochlear development, endocochlear potential generation, active cochlear amplification, and second messenger transduction $[23,24]$.

The GJB2 mutation is one of the most common pathogenic factors related to genetic HL, and GJB2 usually is the first deafness gene to be evaluated during clinical diagnosis due to the observations that GJB2 is the most common human deafness gene in almost all populations studied so far. Currently, more than 300 mutations in GJB2 have been reported (the Human Gene Mutation Database) [25]. Notably, several alleles have been found to be particularly enriched in certain populations: c.35delG in Europe, America, North Africa, and the Middle East; c.71G $>A$ in India and Pakistan; c.167delT in Ashkenazi Jews; and c.109G $>$ A in East and Southeast Asia [16, 26-28]. The contribution of GJB2 mutations to genetic HL varies by ethnicity, but such mutations are the primary cause of congenital severe-toprofound autosomal recessive NSHL (up to $50 \%$ worldwide) $[29,30]$. In addition, mild and moderate HL are associated with GJB2 common mutations such as c.35delG and c.109G $>A$, showing diverse hearing phenotypes [31, 32]. In the Chinese population, the most common GJB2 mutation is c.235delC (68.9\%) [33]. Base deletion creates a frameshift mutation, and early termination of translation yields a nonfunctional protein [34]. Although severe-to-profound HL is the most common clinical presentation of patients with GJB2 c.235delC, various hearing phenotypes have been reported, and the HL caused by the mutation exhibits clinical heterogeneity $[35,36]$.

Understanding genotype-phenotype relationships will provide novel insights into molecular diagnosis, genetic counseling, and genetic therapy. Many approaches, such as cochlear implant surgery, gene therapy, and cell therapy, have been used to treat GJB2-related HL [37-39]. Since 2009, preimplantation genetic diagnosis (PGD), an effective method to prevent the recurrence of genetic HL, has been successfully applied to protect babies against GJB2related HL [40, 41]. We believe that more treatment options will be available for these patients in the future. Here, we analyzed audiological data of 244 patients with GJB2 c.235delC homozygous mutation-induced HL and explored the phenotypic diversity of HL in patients with GJB2 c.235delC with a focus on the symmetry (or lack thereof) of binaural hearing.

\section{Materials and Methods}

2.1. Clinical Data. Patients for whom complete audiological data were available and who visited the molecular diagnostic center of the Chinese PLA General Hospital from 2007 to 2015 were included. The inclusion criteria were (1) binaural SNHL with a complete hearing history, data from physical examination and a detailed ENT examination, and audiological test results; (2) DNA sequence of patients were confirmed to have GJB2 c.235delC homozygous mutation; and (3) no syndromic HL or ear-related diseases (e.g., acute or chronic otitis media, advanced Meniere's disease, acoustic neuroma, meningoencephalitis, or trauma). A total of 244 subjects (130 males and 114 females) were included.

\subsection{Research Methods}

2.2.1. Genomic DNA Extraction. Peripheral blood samples were collected from arm veins, and the genomic DNA of leukocytes was extracted [42].

2.2.2. Detection of Mutations in the Coding Region of GJB2. We performed primer design, PCR amplification, and direct sequencing using the methods descrbied by Dai et al. [43]. We compared the results to the wild-type sequence (GJB2: NM_004004) using GeneTool Lite ver. 1.0.

2.2.3. Audiologic Evaluation. Audiological tests were performed in the hearing center of the Chinese PLA General Hospital. Tests included pure-tone audiometry (or behavioral audiometry) for patients $>4$ years old and multiplefrequency auditory steady-state evoked response (ASSR) tests for patients $\leq 4$ years old.

(1) HL Severity. We derived the average air conduction (AC) pure-tone hearing thresholds or ASSR response thresholds at $0.5,1,2$, and $4 \mathrm{kHz}$ for both ears. If data could not be obtained at any frequency using the maximal output, that output was taken to be the hearing or response threshold. Using the American Speech-Language-Hearing Association (ASHA) [44], we scaled hearing as normal (threshold: $\leq 25 \mathrm{~dB}$ ), mild HL (threshold: 25.1-40 dB), moderate HL (threshold: 40.1-55 dB), moderately severe HL (threshold: 55.1-70 dB), severe HL (threshold: 70.1-90 dB), and profound HL (threshold: $>90 \mathrm{~dB}$, including total deafness). According to WHO criteria [45], disabling HL referred to HL greater than $40 \mathrm{~dB}$ in the better hearing ear in adults and HL greater than 
$30 \mathrm{~dB}$ in the better hearing ear in children (age $\leq 15$ years old). HL progression was defined as an elevation of the average hearing or response thresholds by $>15 \mathrm{~dB}$ in one or both ears between audiograms. Only patients with multiple audiograms and at least a 4-month gap between audiograms were included in the analysis of progressive HL [46].

(2) Audiogram Shapes. We recognized seven shapes of puretone threshold audiograms: descending ( $\geq 15 \mathrm{~dB}$ HL difference between the [better] average thresholds at 250 and $500 \mathrm{~Hz}$ and those at 4,000 and $8,000 \mathrm{~Hz}$ ), flat ( $\leq 15 \mathrm{~dB} \mathrm{HL}$ difference between all thresholds from 125 to $8,000 \mathrm{~Hz}$ ), valley like ( $\geq 10 \mathrm{~dB}$ HL difference between the poorest midfrequency $[1,000-2,000 \mathrm{~Hz}]$ threshold and those at higher and lower frequencies), ascending ( $\geq 15 \mathrm{~dB}$ HL difference between the average thresholds at 250 and $500 \mathrm{~Hz}$ and the [better] average thresholds at 4,000 and $8,000 \mathrm{~Hz}$ ), residual (residual hearing at only one or two frequencies), total deafness (no hearing at any frequency when outputs are maximal), and unclassified (none of the above). We defined descending, flat, valley like, ascending, residual, and total deafness shapes as "regular" audiograms.

We recognized seven shapes of ASSR audiograms: descending ( $\geq 15 \mathrm{~dB}$ HL difference between the [better] mean thresholds at 500 and $1,000 \mathrm{~Hz}$ and those at 2,000 and $4,000 \mathrm{~Hz})$, flat $(\leq 15 \mathrm{~dB} \mathrm{HL}$ difference between all thresholds from 500 to $4,000 \mathrm{~Hz}$ ), valley like ( $\geq 10 \mathrm{~dB} \mathrm{HL}$ difference between the poorest midfrequency $[1,000-2,000 \mathrm{~Hz}]$ threshold and those at higher and lower frequencies), ascending $(\geq 15 \mathrm{~dB}$ HL difference between the mean thresholds at 500 and $1,000 \mathrm{~Hz}$ and the [better] mean thresholds at 2,000 and $4,000 \mathrm{~Hz}$ ), residual (residual hearing at only one or two frequencies), total deafness (no hearing at any frequency when outputs are maximal), and unclassified (none of the above). Similarly, we defined the first six shapes of audiograms as "regular" audiograms.

(3) Asymmetric Hearing Phenotypes. (1) Asymmetry of $H L$ severity (Type A asymmetry): binaural audiograms were shaped similarly, but hearing thresholds differed, with an HL difference $\geq 10 \mathrm{~dB}$ at a minimum of four frequencies, an $\mathrm{HL}$ difference $\geq 15 \mathrm{~dB}$ at two frequencies, or an $\mathrm{HL}$ difference $\geq 25 \mathrm{~dB}$ at one frequency (Figure 1(a)).

(2) Asymmetry of audiogram shape (Type B asymmetry): HL on either side was similar (average hearing threshold difference $\leq 15 \mathrm{~dB}$ ) but audiogram shapes differed, and at least one ear exhibited a regular audiogram (e.g., a descending type in one ear but a flat type in the other; Figure 1(b)).

(3) Asymmetry of HL severity and audiogram shape (Type $C$ asymmetry): the average hearing threshold difference was $>15 \mathrm{~dB}$. In addition, if the audiogram shapes were different, at least one ear exhibited a regular audiogram. If the two audiograms were irregular, the binaural hearing threshold difference was considered asymmetric. Alternatively, if the hearing threshold difference was $\geq 15 \mathrm{~dB}$ at two frequencies from $0.125-8 \mathrm{kHz}$, or $\geq 10 \mathrm{~dB}$ at three frequencies, there was likely Type C asymmetry (Figure 1(c)).

\section{Results}

3.1. Patient Demographics. The patients ranged in age from 3 months to 45 years: 138 patients were $0-4$ years, 60 were 571 years, and 46 were $18-45$ years. Definite ages of onset were identified in 166 patients and ranged from birth to 24 years (average: 1.9 years). Note that six patients were siblings from three families (two per family); the remaining patients were sporadic cases.

3.2. HL Severity. The HL severity (both ears) for all 244 patients is shown in Table 1. Profound HL was most common $(71.93 \%, 351 / 488)$, followed by severe HL $(14.34 \%$, 70/488) and moderately severe HL $(9.43 \%, 46 / 488)$. Fewer patients exhibited moderate HL $(4.10 \%, 20 / 488)$ or mild HL $(0.2 \%, 1 / 488)$. All patients showed disabling HL. Only five patients met the criteria for inclusion in the progressive HL analysis, but none of them had progressive HL.

3.3. Audiograms. Among the 244 cases (488 ears), descending $(30.94 \%, 151 / 488)$ and flat $(24.39 \%, 119 / 488)$ were the most common audiogram shapes, followed by residual $(15.57 \%$, $76 / 488)$, total deafness $(5.53 \%, 27 / 488)$, valley like $(5.33 \%$, $26 / 488)$, and ascending $(2.05 \%, 10 / 488)$. The unclassified shape constituted $16.19 \%$ (79/488) of all audiograms. Table 2 shows that $40.16 \%(98 / 244)$ of cases exhibited the same (regular) audiogram shape in both ears.

3.4. Interaural Hearing Asymmetry. A total of 88 of the 244 cases were symmetric in terms of both HL severity and audiogram shape. By contrast, 156 (63.93\%) cases were asymmetric in terms of audiogram shape and/or HL severity (14 Type A, 106 Type B, and 36 Type C). In the latter group, 211 ears $(67.63 \%)$ exhibited profound HL and 59 (18.91\% of all ears) exhibited severe HL. In patients with symmetric HL, 140 ears (79.55\%) exhibited profound HL (Table 3).

Of the 156 patients with asymmetric interaural HL, 87 ears $(27.88 \%)$ exhibited flat, $69(22.12 \%)$ descending, and $37(11.86 \%)$ residual audiograms. Of the 88 cases with symmetric interaural HL, 82 ears (46.59\%) exhibited descending, $38(21.59 \%)$ residual, and $32(18.18 \%)$ flat audiograms (Table 4).

3.5. HL Severity and Audiograms in Different Age Groups. We categorized age into four groups: group 1 ( $\leq 6$ years old), group 2 (6.1-12 years old), group 3 (12.1-18 years old), and group 4 ( $\geq 18$ years old). There were $156,29,17$, and 42 patients in groups $1,2,3$, and 4 , respectively.

HL severity (both ears) in the different age groups is shown in Table 5. Among all groups, profound HL was the most common type of HL. In groups 1 and 4, severe HL was the second most common type of $\mathrm{HL}$, followed by moderately severe and moderate HL. By contrast, in group 2, moderately severe and moderate HL were the second most common types of HL, followed by severe HL. Similarly, in group 3, moderately severe HL was the second most common type of HL, and a smaller proportion of patients showed moderate and severe HL. However, only one patient in group 1 exhibited mild HL. 


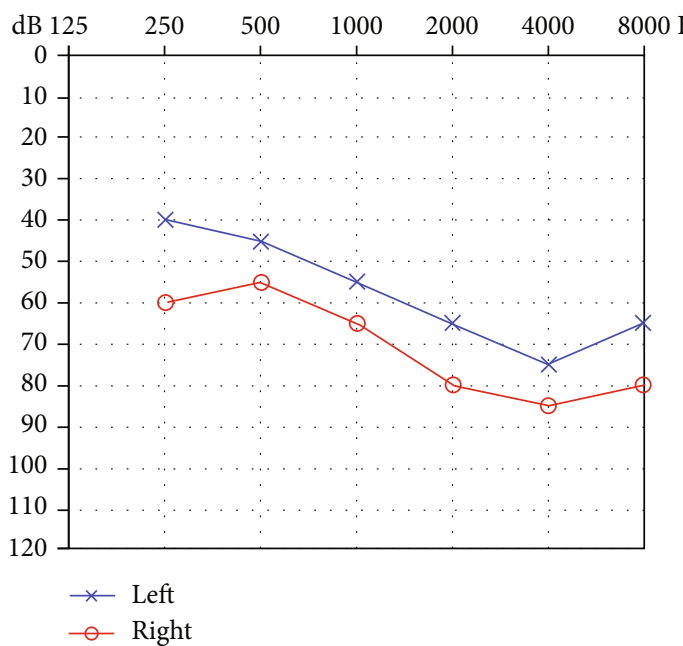

(a)

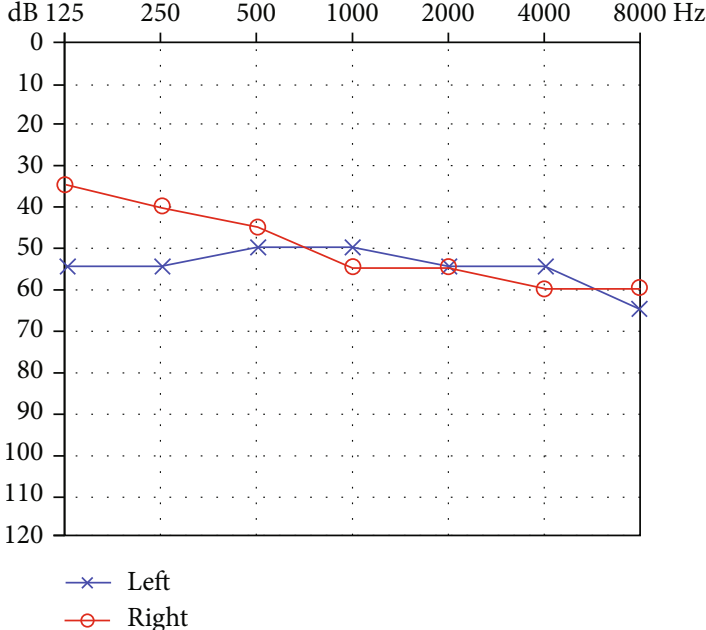

(b)

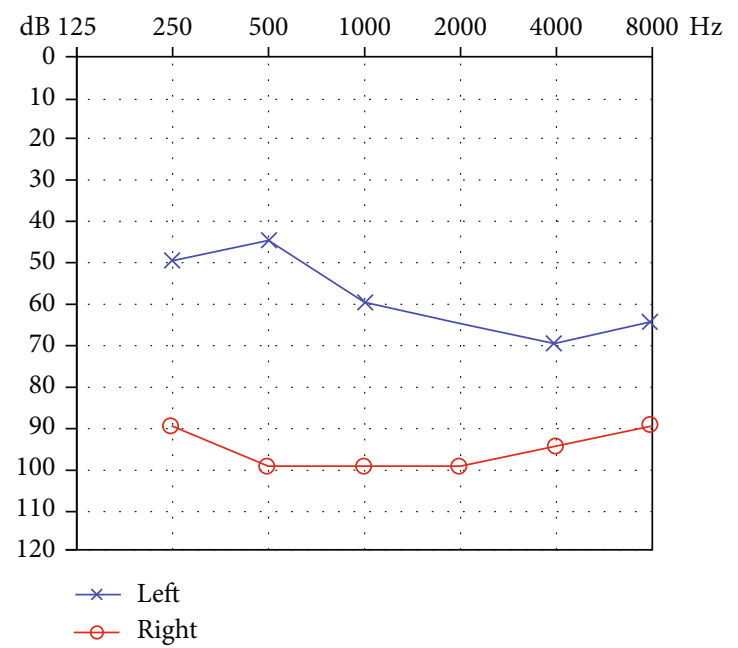

(c)

Figure 1: (a) Audiogram of asymmetry of HL severity (Type A asymmetry). (b) Audiogram of asymmetry of audiogram shape (Type B asymmetry). (c) Audiogram of asymmetry of hearing loss severity and audiogram shape (Type $\mathrm{C}$ asymmetry). In all audiograms, the frequency in hertz $(\mathrm{Hz})$ is plotted on the $x$-axis and the hearing level in decibels $(\mathrm{dB} \mathrm{HL})$ on the $y$-axis.

TABLe 1: HL severity (both ears).

\begin{tabular}{|c|c|c|c|c|c|c|c|}
\hline HL severity & & Mild & Moderate & $\begin{array}{c}\text { Worse ear } \\
\text { Moderate to severe }\end{array}$ & Severe & Profound & Cases \\
\hline \multirow{5}{*}{ Better ear } & Mild & 0 & 0 & 0 & 1 & 0 & 1 \\
\hline & Moderate & & 5 & 7 & 3 & 0 & 15 \\
\hline & Moderate to severe & & & 11 & 10 & 7 & 28 \\
\hline & Severe & & & & 15 & 26 & 41 \\
\hline & Profound & & & & & 159 & 159 \\
\hline
\end{tabular}

Italics indicate patients with the same HL severity in both ears.

The audiograms (both ears) in the different age groups are shown in Table 6 . In group 1 , the most common audiogram shape was flat $(28.53 \%)$. In groups $2-4$, descending was the most common audiogram shape (50\%-61.76\%). Among all groups, total deafness was relatively rare.

The interaural hearing asymmetry in the different age groups is shown in Table 7. Whereas Type B asymmetry
(50.64\%) was the most common type in group 1, symmetry was the most common in groups $2-4(\sim 41-53 \%)$. In groups $1-3$, Type A asymmetry was the least common, whereas in group 4, Type $\mathrm{C}$ asymmetry was the least common.

3.6. HL in Three Sets of Siblings. Set 1 was composed of two sisters (Figure 2(a)). Their normal-hearing parents were 
TABLE 2: Audiogram shapes of both ears.

\begin{tabular}{|c|c|c|c|c|c|c|c|c|}
\hline \multirow{2}{*}{\multicolumn{2}{|c|}{ Audiogram shape }} & \multicolumn{7}{|c|}{ The other ear } \\
\hline & & Descending & Flat & Residual & Valley like & Ascending & Total deafness & Other \\
\hline \multirow{7}{*}{ One ear } & Descending & 50 & 21 & 8 & 6 & 1 & 2 & 13 \\
\hline & Flat & & 21 & 12 & 8 & 4 & 3 & 29 \\
\hline & Residual & & & 19 & 1 & 0 & 5 & 12 \\
\hline & Valley like & & & & 1 & 1 & 1 & 7 \\
\hline & Ascending & & & & & 1 & 0 & 2 \\
\hline & Total deafness & & & & & & 6 & 4 \\
\hline & Other & & & & & & & 6 \\
\hline
\end{tabular}

Italics indicate patients with the same audiogram shape in both ears.

TABLE 3: HL severity in patients with asymmetric and symmetric interaural HL.

\begin{tabular}{|c|c|c|c|c|c|c|c|}
\hline HL severity & & Mild & Moderate & $\begin{array}{c}\text { Worse ear } \\
\text { Moderate to severe }\end{array}$ & Severe & Profound & Cases \\
\hline \multirow{5}{*}{ Better ear } & Mild & $0(0)$ & $0(0)$ & $0(0)$ & $0(1)$ & $0(0)$ & $0(1)$ \\
\hline & Moderate & & $4(1)$ & $4(3)$ & $0(3)$ & $0(0)$ & $8(7)$ \\
\hline & Moderate to severe & & & $4(7)$ & $1(9)$ & $0(7)$ & $5(23)$ \\
\hline & Severe & & & & $4(11)$ & $2(24)$ & $6(35)$ \\
\hline & Profound & & & & & $69(90)$ & $69(90)$ \\
\hline
\end{tabular}

(1) The value outside/inside each pair of parentheses represents the number of patients with symmetric/asymmetric interaural HL. (2) Italics indicate patients with the same HL severity in both ears.

TABLE 4: Audiogram shapes in patients with asymmetric and symmetric interaural HL.

\begin{tabular}{|c|c|c|c|c|c|c|c|c|}
\hline \multicolumn{2}{|c|}{ Audiogram shape } & \multicolumn{7}{|c|}{ The other ear } \\
\hline \multirow{7}{*}{ One ear } & Descending & $41(9)$ & $0(21)$ & $0(8)$ & $0(6)$ & $0(1)$ & $0(2)$ & $0(13)$ \\
\hline & Flat & & $16(5)$ & $0(12)$ & $0(8)$ & $0(4)$ & $0(3)$ & $0(29)$ \\
\hline & Residual & & & $19(0)$ & $0(1)$ & $0(0)$ & $0(5)$ & $0(12)$ \\
\hline & Valley like & & & & $1(0)$ & $0(1)$ & $0(1)$ & $0(7)$ \\
\hline & Ascending & & & & & $0(1)$ & $0(0)$ & $0(2)$ \\
\hline & Total deafness & & & & & & $6(0)$ & $0(4)$ \\
\hline & Other & & & & & & & $5(1)$ \\
\hline
\end{tabular}

(1) The value outside/inside each pair of parentheses represents the number of patients with symmetric/asymmetric interaural HL. (2) Italics indicate patients with the same audiogram shape for both ears.

TABLE 5: Degree of hearing loss in patients (both ears) according to age group.

\begin{tabular}{|c|c|c|c|c|c|}
\hline Age & Mild & Moderate & $\begin{array}{l}\text { Degree of hearing los } \\
\text { Moderate to severe }\end{array}$ & Severe & Profound \\
\hline Group 1 & $1(0.32 \%)$ & $2(0.64 \%)$ & $19(6.09 \%)$ & $42(13.46 \%)$ & $248(79.49 \%)$ \\
\hline Group 2 & 0 & $12(20.69 \%)$ & $12(20.69 \%)$ & $9(15.52 \%)$ & $25(43.10 \%)$ \\
\hline Group 3 & 0 & $4(11.76 \%)$ & $8(23.53 \%)$ & $4(11.76 \%)$ & $18(52.94 \%)$ \\
\hline Group 4 & 0 & $2(2.38 \%)$ & $7(8.33 \%)$ & $15(17.86 \%)$ & $60(71.43 \%)$ \\
\hline
\end{tabular}

Group 1 ( $\leq 6$ years old), group 2 (6.1-12 years old), group 3 (12.1-18 years old), and group 4 ( $\geq 18$ years old). The value outside each pair of parentheses represents the number of ears with this degree of hearing loss; the value inside each pair of parentheses represents the percentage of ears with this degree of hearing loss relative to the total number of ears in each group.

heterozygous carriers of c.235delC. The older sister (II-1) suffered from moderate and severe HL in the left and right ears. The younger sister (II-2) suffered from binaural moderate HL. The audiograms of the older sister were descending and unclassified (one ear each). The audiograms of the younger sister were flat in both ears. The older sister exhibited Type $\mathrm{C}$ asymmetric HL, and the younger sister exhibited symmetric HL. Set 2 was composed of two brothers 
TABLE 6: Types of audiograms in patients (both ears) with different age groups.

\begin{tabular}{|c|c|c|c|c|c|c|c|}
\hline \multirow{2}{*}{ Age } & \multicolumn{7}{|c|}{ Types of audiograms } \\
\hline & Descending & Flat & Residual & Valley like & Ascending & Total deafness & Other \\
\hline Group 1 & $56(17.95 \%)$ & $89(28.53 \%)$ & $53(16.99 \%)$ & $20(6.41 \%)$ & $7(2.24 \%)$ & $23(7.37 \%)$ & $64(20.51 \%)$ \\
\hline Group 2 & $32(55.17 \%)$ & $11(18.97 \%)$ & $2(3.45 \%)$ & $5(8.62 \%)$ & $2(3.45 \%)$ & 0 & $6(10.34 \%)$ \\
\hline Group 3 & $21(61.76 \%)$ & $5(14.71 \%)$ & $3(8.82 \%)$ & 0 & 0 & $1(2.94 \%)$ & $4(11.76 \%)$ \\
\hline Group 4 & $42(50.00 \%)$ & $14(16.67 \%)$ & $17(20.24 \%)$ & $1(11.90 \%)$ & $1(11.90 \%)$ & $3(3.57 \%)$ & $6(7.14 \%)$ \\
\hline
\end{tabular}

Group 1 ( $\leq 6$ years old), group 2 (6.1-12 years old), group 3 (12.1-18 years old), and group 4 ( $\geq 18$ years old). The value outside each pair of parentheses represents the number of ears with this type of audiogram; the value inside each pair of parentheses represents the percentage of ears with this type of audiogram relative to the total number of ears in each group.

TABLE 7: Interaural hearing symmetry or asymmetry in patients according to age group.

\begin{tabular}{lcccc}
\hline \multirow{4}{*}{ Age } & \multicolumn{4}{c}{ Interaural hearing symmetry or asymmetry } \\
& Symmetry & Type A & Typyme B & Type C \\
\hline Group 1 & $47(30.13 \%)$ & $7(4.49 \%)$ & $79(50.64 \%)$ & $23(14.74 \%)$ \\
Group 2 & $12(41.38 \%)$ & $1(3.45 \%)$ & $10(34.48 \%)$ & $6(20.69 \%)$ \\
Group 3 & $9(52.94 \%)$ & 0 & $6(35.29 \%)$ & $2(11.76 \%)$ \\
Group 4 & $20(47.62 \%)$ & $6(14.29 \%)$ & $11(26.19 \%)$ & $5(11.90 \%)$ \\
\hline
\end{tabular}

Group 1 ( $\leq 6$ years old), group 2 (6.1-12 years old), group 3 (12.1-18 years old), and group 4 ( $\geq 18$ years old). The value outside each pair of parentheses represents the number of patients with interaural hearing symmetry or asymmetry; the value inside each pair of parentheses represents the percentage of patients with interaural hearing symmetry or asymmetry relative to the total number of patients in each group.

(Figure 2(b)). Their normal-hearing parents were heterozygous carriers of c.235delC. Both brothers (II-1 and II-2) presented with binaural profound HL. The audiograms of the elder brother were descending in both ears. The audiograms of the younger brother were descending and flat (one ear each). The elder brother exhibited symmetric HL, and the younger brother exhibited Type B asymmetric HL. Set 3 was composed of an older sister and a younger brother (Figure 2(c)). Their parents and sister were all heterozygous carriers of c.235delC and had no hearing problems. Both siblings (II-1 and II-3) exhibited similar binaural moderate to severe HL with descending and flat audiograms (one of each). Both exhibited Type B asymmetric HL (Figure 3 and Table 8).

\section{Discussion}

HL has become a global public health problem. In addition to impaired communication, HL is associated with language delays, social adaptation problems, and even dementia [47]. Hereditary SNHL is genetically heterogeneous, and pathogenic mutations have been identified in approximately 50$60 \%$ of cases [48]. GJB2 mutation is a major cause of hereditary NSHL, and most mutations are located in the coding region [49]. Up to $50 \%$ of cases of autosomal recessive NSHL are attributable to GJB2 mutation in many populations worldwide [29, 30]. Therefore, genetic testing for GJB2 mutations is a primary screening process for the molecular diagnosis of HL. As we mentioned previously, the GJB2 mutation spectrum varies ethnically and geographically. In the Chinese population, the c.235delC homozygous mutation is the most common mutation in GJB2 [33]. This gene encodes the connexin $26(\mathrm{Cx} 26)$ protein. The gap junction proteins of adjacent cells allow for the exchange of information and materials; electrolytes, second messengers, and metabolites move through these channels, underlying both intercellular communication and homeostasis of the cochlear fluids, endolymph, and perilymph [50,51]. Mutations in the GJB2 coding region can cause frameshifts affecting protein translation and gap junction protein structure, resulting in a defective protein [52]. The GJB2 c.235delC mutation causes early termination of translation and produces a nonfunctional $\mathrm{Cx} 26$ protein. The null $\mathrm{Cx} 26$ can induce apoptosis and oxidative damage in the cochlear duct, reduce the release of glutathione from connexin hemichannels, and decrease nutrient delivery to the sensory epithelium via cochlear gap junctions, thereby leading to HL. Cx26-deficient mouse models showed congenital HL and cochlear developmental disorders [53-55]. GJB2-related HL is usually binaural [42, 56], as in all 244 patients in this study. Profound HL was the most common $(71.93 \%, 351 / 488) \mathrm{HL}$ in our patients, followed by severe $(14.34 \%, 70 / 488)$, moderately severe (9.43\%, 46/488), and moderate $(4.10 \%, 20 / 488)$ HL. Mild HL was seen in only $0.2 \%(1 / 488)$ of cases. Zhao et al. [35] retrospectively analyzed the hearing phenotypes of a large group of Chinese patients with HL caused by GJB2 c.235delC biallelic mutations and found that most patients exhibited severe or profound HL, with only a few showing moderate HL. We also found that profound HL was most common; however, a considerable proportion of our cases exhibited moderate $(4.10 \%)$ or moderately severe $(9.43 \%)$ HL. HL phenotypes thus differ. All patients in our study showed disabling HL, indicating that GJB2-related HL usually has a negative impact on quality of life, ability to listen in noisy environments, communication with others, and comprehension ability, regardless of whether hearing in the two ears is symmetric. In the analysis of progressive HL, all five patients who met the criteria showed stabilization of HL. Our results were consistent with those of Chorath et al. [46], who concluded that progression of GJB2-related HL was rare. Unfortunately, the number of patients included was limited, and more follow-up data are required in future studies. Some researchers use the following definitions to classify audiograms $[57,58]$ : descending $(>15 \mathrm{~dB}$ HL difference between the [better] average thresholds at 500 and $1,000 \mathrm{~Hz}$ and those 


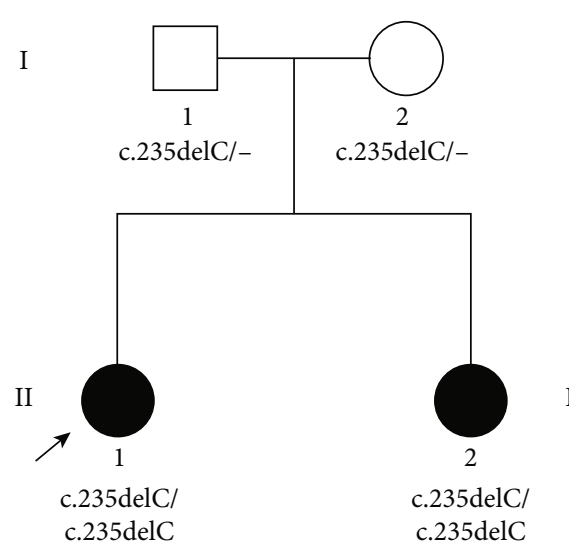

(a)

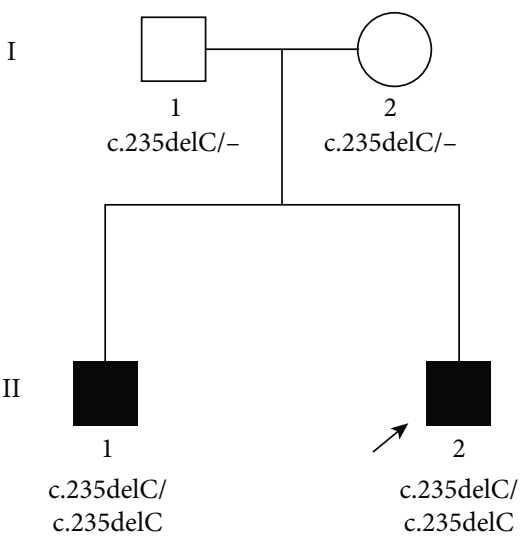

(b)

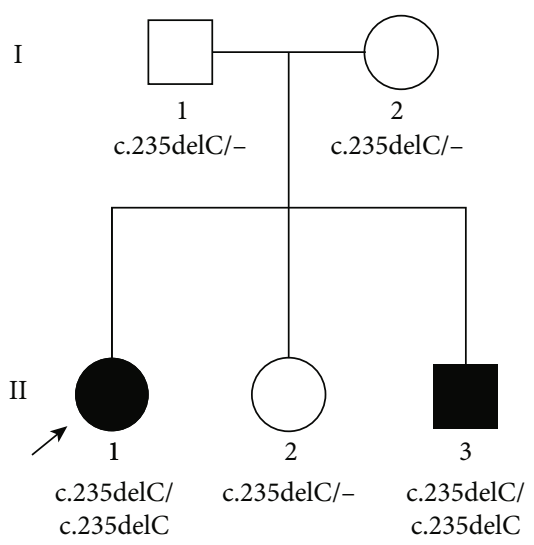

(c)

Figure 2: $(\mathrm{a}-\mathrm{c})$ are genotypes of the pedigrees for sets 1,2 , and 3, respectively.

at 4,000 and $8,000 \mathrm{~Hz})$, flat $(<15 \mathrm{~dB} \mathrm{HL}$ difference between all thresholds from 250 to $8,000 \mathrm{~Hz}$ ), midfrequency U-shaped ( $>15 \mathrm{~dB}$ HL difference between the poorest midfrequency $[1,000-2,000 \mathrm{~Hz}]$ thresholds and those of higher and lower frequencies), and ascending (>15 dB HL difference between the average thresholds at 500 and $1,000 \mathrm{~Hz}$ and the [better] thresholds at 4,000 and $8,000 \mathrm{~Hz}$ ). However, in our clinic, we found that the results yielded using these definitions sometimes do not agree with the characteristics of the audiograms. Thus, we amended the definitions. First, we changed the difference in $\mathrm{dB}$ HL from $>15 \mathrm{~dB}$ to $\geq 15 \mathrm{~dB}$ for both the descending and ascending types, from $<15 \mathrm{~dB}$ to $\leq 15 \mathrm{~dB}$ for the flat type, and from $>15 \mathrm{~dB}$ to $\geq 10 \mathrm{~dB}$ for the valley-like type. Second, we used 250 and $500 \mathrm{~Hz}$ (not 500 and $1,000 \mathrm{~Hz}$ ) as the low frequencies for the descending and ascending types and 2,000 and $4,000 \mathrm{~Hz}$ (not 4,000 and $8,000 \mathrm{~Hz}$ ) as the high frequencies when evaluating ASSR data. In addition, when defining the flat type of ASSR, we use frequencies from 500 to $4,000 \mathrm{~Hz}$. Third, we consider the residual and total deafness types to be "regular" types. We found that the descending (30.94\%) and flat $(24.39 \%)$ types were the most common, followed by the residual (15.57\%), total deafness (5.53\%), valley-like (5.33\%), and ascending $(2.05 \%)$ types. Our findings are similar to those of King et al. [57] in that most audiograms were descending, flat, or residual.
The phenotypes of genetic HL remain poorly understood. We analyzed the audiograms of 244 cases with homozygous GJB2 c.235delC-associated HL. No consensus definition of "asymmetry" in the context of binaural HL has emerged. Early studies [59-61] proposed that an interaural difference(s) in pure-tone thresholds $\geq 10 \mathrm{~dB}$ at two frequencies or $\geq 15 \mathrm{~dB}$ at one frequency constitutes asymmetric HL. In a 2007 audiogram classification system (AMCLASS) [62, 63], audiograms were considered asymmetric if at least three frequencies differed by $\geq 10 \mathrm{~dB}$, two by $\geq 15 \mathrm{~dB}$, or one by $\geq 20 \mathrm{~dB}$ over the range of $0.25-8 \mathrm{kHz}$. In 2009, Mazzoli et al. [64] defined asymmetric HL as differences $>10 \mathrm{~dB}$ for at least two frequencies. These criteria concern only the severity of $\mathrm{HL}$, not the audiogram shape; the picture is thus incomplete. To better characterize asymmetry, we divided it into three types with reference to both audiogram shape and HL severity. A substantial proportion of our cases with binaural HL exhibited interaural asymmetry in terms of audiogram shape and/or HL severity. A total of 156 (63.93\%) patients exhibited asymmetric HL: 14 in terms of HL severity (Type A), 106 in terms of audiogram shape (Type B), and 36 with both Type A and B features (Type C). Our figures differ significantly from those of Wang et al. [36], who found a rate of asymmetric HL of $37.37 \%$ in children with biallelic protein-truncating mutations. This difference is probably attributable to variation in the definitions of binaural asymmetric HL. It is clear, 

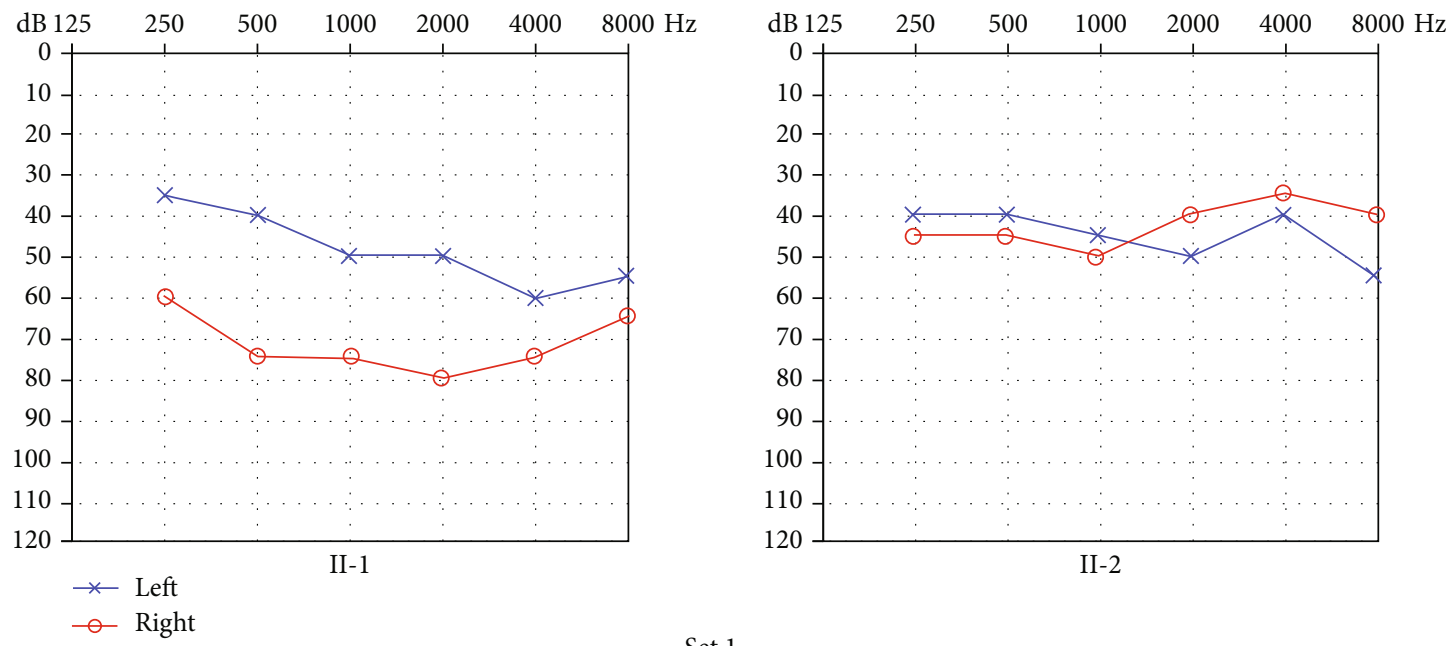

Set 1

(a) Set 1
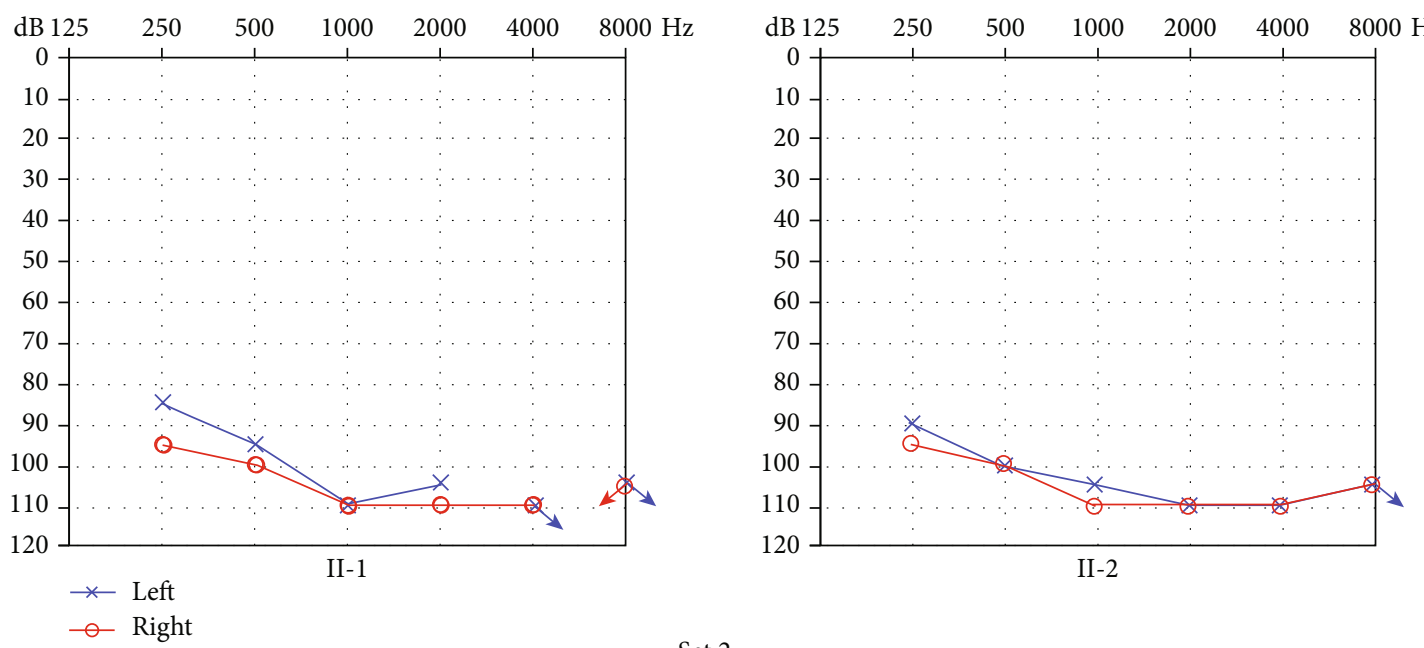

Set 2

(b) Set 2
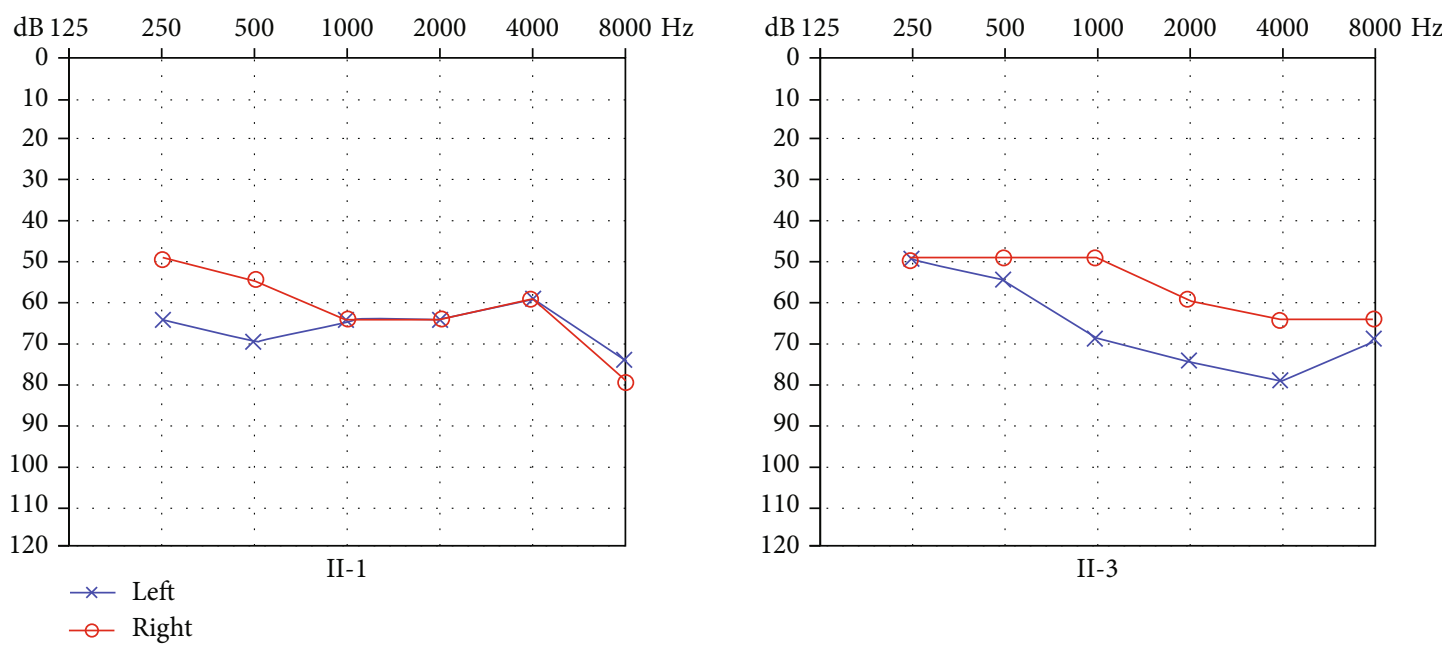

Set 3

(c) Set 3

Figure 3: (a) Audiograms of two sisters (II-1 and II-2) in set 1. (b) Audiograms of two brothers (II-1 and II-2) in set 2. (c) Audiograms of the older sister (II-1) and younger brother (II-3) in set 3 . 
TABLE 8: HL severity and audiogram shapes in three pairs of siblings.

\begin{tabular}{cccccc}
\hline & & & HL severity & & \multicolumn{2}{c}{ Audiogram shape } \\
& & Left ear & Right ear & Left ear & Right ear \\
\hline \multirow{2}{*}{ Set 1} & Older sister & Moderate & Severe & Descending & Other \\
& Younger sister & Moderate & Moderate & Flat & Flat \\
Set 2 & Older brother & Profound & Profound & Descending & Descending \\
& Younger brother & Profound & Profound & Descending & Flat \\
\multirow{2}{*}{ Set 3} & Older sister & Moderate to severe & Moderate to severe & Flat & Descending \\
& Younger brother & Moderate to severe & Moderate to severe & Descending & Flat \\
\hline
\end{tabular}

however, that the GJB2 c.235delC mutation is associated with significant variation in the binaural HL phenotype, evidencing a high level of genetic heterogeneity.

Among our 156 patients exhibiting interaural HL asymmetry, 211 ears (67.63\%) suffered profound HL and 59 (18.91\%) suffered severe HL. By contrast, in the 88 cases evidencing symmetric interaural HL, 140 ears (79.55\%) showed profound HL. Thus, HL in such patients is likely to be profound or severe regardless of symmetry or asymmetry. It is worth noting that in patients with symmetric interaural HL, daily communication with others will be challenging since most suffered from bilateral profound HL. Among patients with asymmetric HL, audiograms were (in order) flat (27.88\%), descending (22.12\%), and residual (11.86\%). By contrast, among patients with symmetric HL, audiograms were (in order) descending (46.59\%), residual (21.59\%), and flat (18.18\%). Such subtle differences may be attributable to variation in HL severity. In all groups of different ages, profound HL was most common. HL severity tended to be more serious in group 1 ( $\leq 6$ years old) and group $4(\geq 18$ years old). Among the patients $<18$ years old, the most common audiogram shapes were descending and flat, whereas among the adult patients, the most common audiogram shapes were descending and residual. The differences may also be attributable to variation in HL severity. Among patients $\leq 6$ years old, Type B asymmetry was the most common. However, among patients $>6$ years old, symmetry was the most common. In three sibling pairs, HL severity was similar but audiogram shapes differed. Asymmetry may be attributable to one or more of heredity, epigenetics, and/or the environment. However, the mechanism of GJB2-related HL remains unclear. More clinical data, combined with full-exome and whole-genome sequencing, are needed.

\section{Conclusion}

A considerable proportion of patients homozygous for the GJB2 c.235delC mutation exhibit significant variation in their binaural HL phenotypes, reflecting a high degree of bilateral HL asymmetry.

\section{Data Availability}

The data used to support the findings of this study are available from the corresponding authors upon request.

\section{Ethical Approval}

This study was approved by the Ethics Committee of the Chinese People's Liberation Army General Hospital (reference number S2016-120-02).

\section{Disclosure}

The funders had no role in study design, data collection and analysis, decision to publish, or preparation of the manuscript.

\section{Conflicts of Interest}

The authors have no conflicts of interest to declare.

\section{Authors' Contributions}

Chang Guo and Sha-Sha Huang contributed equally to this work.

\section{Acknowledgments}

We sincerely thank all the family members for their participation and cooperation in this study. This study was supported by grants from the National Key R\&D Project (2016YFC1000700, 2016YFC1000704), National Natural Science Foundation of China $(81730029,61827805)$, and Beijing Natural Science Foundation (7191011) to Pu Dai; grants from the Beijing Natural Science Foundation (7192234) and National Natural Science Foundation of China (81570929) to Xue Gao; a grant from the National Natural Science Foundation of China (81870731) to Sha-Sha Huang; and grants from the National Key R\&D Project (2016YFC1000706), National Natural Science Foundation of China (81873704), and Fostering Funds of Chinese PLA General Hospital for National Distinguished Young Scholar Science Fund (2017JQPY-001) to Yong-Yi Yuan.

\section{References}

[1] S. Ahmadmehrabi, J. Brant, D. J. Epstein, M. J. Ruckenstein, and D. J. Rader, "Genetics of postlingual sensorineural hearing Loss," The Laryngoscope, pp. 1-9, 2020.

[2] "Deafness and hearing loss," March 2020, https://www.who .int/newsroom/fact-sheets/detail/deafness-and-hearing-loss. 
[3] Y. Liu, J. Qi, X. Chen et al., "Critical role of spectrin in hearing development and deafness," Science Advances, vol. 5, no. 4, 2019.

[4] J. Qi, Y. Liu, C. Chu et al., “A cytoskeleton structure revealed by super-resolution fluorescence imaging in inner ear hair cells," Cell Discovery, vol. 5, no. 1, 2019.

[5] R. Beach, J. M. Abitbol, B. L. Allman, J. L. Esseltine, Q. Shao, and D. W. Laird, "GJB2 mutations linked to hearing loss exhibit differential trafficking and functional defects as revealed in cochlear-relevant cells," Frontiers in Cell and Developmental Biology, vol. 8, 2020.

[6] S. Zhang, Y. Zhang, Y. Dong et al., "Knockdown of Foxg1 in supporting cells increases the trans-differentiation of supporting cells into hair cells in the neonatal mouse cochlea," Cellular and Molecular Life ences, vol. 77, no. 7, pp. 1401-1419, 2020.

[7] C. Cheng, Y. Wang, L. Guo et al., "Age-related transcriptome changes in Sox2+ supporting cells in the mouse cochlea," Stem Cell Research \& Therapy, vol. 10, no. 1, p. 365, 2019.

[8] S. Zhang, D. Liu, Y. Dong et al., "Frizzled-9+ supporting cells are progenitors for the generation of hair cells in the postnatal mouse cochlea," Frontiers in Molecular Neuroence, vol. 12, p. 184, 2019.

[9] Z. He, L. Guo, Y. Shu et al., "Autophagy protects auditory hair cells against neomycin-induced damage," Autophagy, vol. 13, no. 11, pp. 1884-1904, 2017.

[10] W. Liu, X. Xu, Z. Fan, G. Sun, Y. Han, and D. Zhang, "Wnt signaling activates TIGAR and protects against cisplatin-induced spiral ganglion neuron damage in the mouse cochlea," Antioxidants \& Redox Signaling, vol. 30, no. 11, pp. 1389-1410, 2019.

[11] Z.-h. He, S.-y. Zou, M. Li et al., "The nuclear transcription factor Foxg1 affects the sensitivity of mimetic aging hair cells to inflammation by regulating autophagy pathways," Redox Biology, vol. 28, article 101364, 2020.

[12] K. W. Chang, "Genetics of Hearing Loss-Nonsyndromic," Otolaryngologic Clinics of North America, vol. 48, no. 6, pp. 1063-1072, 2015.

[13] H. K. Kurtulgan, E. E. Altuntaş, M. E. Yıldırım, Ö. Özdemir, B. Bağc1, and İ. Sezgin, "The analysis of GJB2, GJB3, and GJB6 gene mutations in patients with hereditary nonsyndromic hearing loss living in Sivas," The Journal of International Advanced Otology, vol. 15, no. 3, pp. 373-378, 2019.

[14] M. Koohiyan, A. Ahmadi, F. Koohian, S. Aghaei, B. Amiri, and M. Hashemzadeh-Chaleshtorica, "An update of spectrum and frequency of GJB2 mutations causing hearing loss in the south of Iran: A literature review," International Journal of Pediatric Otorhinolaryngology, vol. 119, pp. 136-140, 2019.

[15] X. Yu, Y. Lin, J. Xu et al., "Molecular epidemiology of Chinese Han deaf patients with bi-allelic and mono-allelic GJB2 mutations," Orphanet Journal of Rare Diseases, vol. 15, no. 1, p. 29, 2020.

[16] M. Koohiyan, F. Azadegan-Dehkordi, F. Koohian, and M. Hashemzadeh-Chaleshtori, "Genetics of hearing loss in North Iran population: an update of spectrum and frequency of GJB2 mutations," Journal of Audiology \& Otology, vol. 23, no. 4, pp. 175-180, 2019.

[17] J. Shinagawa, H. Moteki, S.-y. Nishio, Y. Noguchi, and S.i. Usami, "Haplotype analysis of GJB2 mutations: founder effect or mutational hot spot?," Genes, vol. 11, no. 3, p. 250, 2020.

[18] M. Kabra, P. K. Singh, S. Sharma, M. Ghosh, S. S. Shastri, and N. Gupta, "Spectrum of GJB2 gene variants in Indian children with non-syndromic hearing loss," The Indian Journal of Medical Research, vol. 147, no. 6, pp. 615-618, 2018.

[19] S. Maeda, S. Nakagawa, M. Suga et al., "Structure of the connexin 26 gap junction channel at $3.5 \AA$ resolution," Nature, vol. 458, no. 7238, pp. 597-602, 2009.

[20] H. Xia, X. Huang, H. Xu et al., "GJB2 c.235delC variant associated with autosomal recessive nonsyndromic hearing loss and auditory neuropathy spectrum disorder," Genetics and Molecular Biology, vol. 42, no. 1, pp. 48-51, 2019.

[21] H. Jiang, Y. Niu, L. Qu, X. Huang, X. Zhu, and G. Tang, “A novel compound heterozygous mutation in the GJB2 gene is associated with non-syndromic hearing loss in a Chinese family," Bioscience Trends, vol. 12, no. 5, pp. 470-475, 2018.

[22] F. Mammano, "Inner ear connexin channels: roles in development and maintenance of cochlear function," Cold Spring Harbor Perspectives in Medicine, vol. 9, no. 7, 2019.

[23] L. Mei, J. Chen, L. Zong et al., "A deafness mechanism of digenic Cx26 (GJB2) and Cx30 (GJB6) mutations: Reduction of endocochlear potential by impairment of heterogeneous gap junctional function in the cochlear lateral wall," Neurobiology of Disease, vol. 108, pp. 195-203, 2017.

[24] X. Lin, X. Lin, G. Li et al., "Hearing consequences in GJB2 knock-in mice: implications for human p.V37I mutation," Aging, vol. 11, no. 18, pp. 7416-7441, 2019.

[25] http://www.hgmd.cf.ac.uk/.

[26] H. Kaheel, A. Breß, M. A. Hassan et al., "Frequency of c.35delG mutation in GJB2 gene (connexin 26) in Syrian patients with nonsyndromic hearing impairment," Genetics Research International, vol. 2017, 3 pages, 2017.

[27] H. Shaikh, A. M. Waryah, A. K. Narsani et al., "Genetic testing of non-familial deaf patients for CIB2 and GJB2 mutations: phenotype and genetic counselling," Biochemical Genetics, vol. 55, no. 5-6, pp. 410-420, 2017.

[28] M. H. Bouzaher, C. P. Worden, and A. Jeyakumar, "Systematic review of pathogenic GJB2 variants in the Latino population," Otology \& Neurotology, vol. 41, p. 2, 2019.

[29] N. Kecskeméti, M. Szönyi, A. Gáborján et al., "Analysis of GJB2 mutations and the clinical manifestation in a large Hungarian cohort," European Archives of Oto-Rhino-Laryngology, vol. 275, no. 10, pp. 2441-2448, 2018.

[30] A. M. Sheffield and R. J. H. Smith, "The epidemiology of deafness," Cold Spring Harbor Perspectives in Medicine, vol. 9, no. 9, 2019.

[31] J.-C. Leclère, M.-S. Le Gac, C. Le Maréchal, C. Ferec, and R. Marianowski, "GJB2 mutations: Genotypic and phenotypic correlation in a cohort of 690 hearing-impaired patients, toward a new mutation?," International Journal of Pediatric Otorhinolaryngology, vol. 102, pp. 80-85, 2017.

[32] Y. Chai, D. Chen, L. Sun et al., "The homozygous p.V37I variant of GJB2 is associated with diverse hearing phenotypes," Clinical Genetics, vol. 87, no. 4, pp. 350-355, 2015.

[33] P. Dai, F. Yu, B. Han et al., "GJB2 mutation spectrum in 2063 Chinese patients with nonsyndromic hearing impairment," Journal of Translational Medicine, vol. 7, no. 1, p. 26, 2009.

[34] F. Yu, P. Dai, and D. Y. Han, "GJB2 gene mutation and prelingual hereditary non-syndromic deafness," Otolaryngology Foreign Medical Sciences, vol. 29, no. 6, pp. 359-361, 2005.

[35] F.-F. Zhao, Y.-B. Ji, D.-Y. Wang et al., "Phenotype-genotype correlation in 295 Chinese deaf subjects with biallelic causative mutations in the GJB2 Gene," Genetic Testing \& Molecular Biomarkers, vol. 15, no. 9, pp. 619-625, 2011. 
[36] X. Wang, L. Huang, X. Zhao et al., "Children with GJB2 gene mutations have various audiological phenotypes," Bioscience Trends, vol. 12, no. 4, pp. 419-425, 2018.

[37] B. György, C. Sage, A. A. Indzhykulian et al., "Rescue of Hearing by Gene Delivery to Inner-Ear Hair Cells Using ExosomeAssociated AAV," Molecular Therapy, vol. 25, no. 2, pp. 379391, 2017.

[38] R. Mittal, D. Nguyen, A. P. Patel et al., "Recent advancements in the regeneration of auditory hair cells and hearing restoration," Frontiers in Molecular Neuroence, vol. 10, 2017.

[39] X. Gao, Y. Tao, V. Lamas et al., "Treatment of autosomal dominant hearing loss by in vivo delivery of genome editing agents," Nature, vol. 553, no. 7687, pp. 217-221, 2018.

[40] G. Altarescu, T. Eldar-Geva, B. Brooks et al., "Preimplantation genetic diagnosis (PGD) for nonsyndromic deafness by polar body and blastomere biopsy," Journal of Assisted Reproduction and Genetics, vol. 26, no. 7, pp. 391-397, 2009.

[41] W. P. Xiong, D. Y. Wang, Y. Gao et al., "Reproductive management through integration of PGD and MPS-based noninvasive prenatal screening/diagnosis for a family with GJB2-associated hearing impairment," Science China Life Sciences, vol. 58, no. 9, pp. 829-838, 2015.

[42] Z. Y. Dai, B. C. Sun, S. S. Huang et al., "Audiological features/genotype correlations in GJB2 mutation," Chinese Journal of Otology, vol. 1, pp. 34-36, 2014.

[43] P. Dai, F. Yu, D.-y. Kang et al., "Diagnostic methods and clinic application for mtDNA A1555G and GJB2 and SLC26A4 genes in deaf patients," Chinese Journal of Otorhinolaryngology Head and Neck Surgery, vol. 40, no. 10, pp. 769-773, 2005.

[44] T. Koffler, K. Ushakov, and K. B. Avraham, "Genetics of Hearing Loss: Syndromic," Otolaryngologic Clinics of North America, vol. 48, no. 6, pp. 1041-1061, 2015.

[45] https://www.who.int/news-room/fact-sheets/detail/deafnessand-hearing-loss.

[46] K. H. Lee, D. A. Larson, G. Shott et al., "Audiologic and temporal bone imaging findings in patients with sensorineural hearing loss and GJB2 mutations," Laryngoscope, vol. 119, no. 3, pp. 554-558, 2009.

[47] K. T. Chorath, M. J. Willis, N. Morton-Gonzaba, W. J. Humann, and A. Moreira, "Mesenchymal stem cells for sensorineural hearing loss: protocol for a systematic review of preclinical studies," Systematic Reviews, vol. 8, no. 1, p. 126, 2019.

[48] M. M. Cohen and R. J. Gorlin, "Epidemiology, etiology and genetic patterns," in Hereditary Hearing Loss and its Syndromes, R. J. Gorlin, H. V. Toriello, and M. M. Cohen, Eds., pp. 9-21, Oxford University Press, Oxford, 1995.

[49] G. Lucotte and F. Diéterlen, "The 35delG mutation in the connexin 26 gene (GJB2) associated with congenital deafness: European carrier frequencies and evidence for its origin in ancient Greece," Genetic Testing, vol. 9, no. 1, pp. 20-25, 2005.

[50] I. Todt, H. C. Hennies, D. Basta, and A. Ernst, "Vestibular dysfunction of patients with mutations of connexin 26," Neuroreport, vol. 16, no. 11, pp. 1179-1181, 2005.

[51] S. L. Johnson, F. Ceriani, O. Houston et al., "Connexin-mediated signaling in nonsensory cells is crucial for the development of sensory inner hair cells in the mouse cochlea," The Journal of Neuroscience, vol. 37, no. 2, pp. 258-268, 2017.

[52] M. Blanchard, B. Thierry, S. Marlin, and F. Denoyelle, "Genetic aspects of congenital sensorineural hearing loss," Archives de Pédiatrie, vol. 19, no. 8, pp. 886-889, 2012.
[53] J. Chen, J. Chen, Y. Zhu, C. Liang, and H.-B. Zhao, "Deafness induced by Connexin 26 (GJB2) deficiency is not determined by endocochlear potential (EP) reduction but is associated with cochlear developmental disorders," Biochemical and Biophysical Research Communications, vol. 448, no. 1, pp. 28-32, 2014.

[54] H. B. Zhao, "Hypothesis of K+-recycling defect is not a primary deafness mechanism for Cx26 (GJB2) deficiency," Frontiers in Molecular Neuroence, vol. 10, no. 126, 2017.

[55] A. R. Fetoni, V. Zorzi, F. Paciello et al., "Cx26 partial loss causes accelerated presbycusis by redox imbalance and dysregulation of Nfr2 pathway," Redox Biology, vol. 19, pp. 301-317, 2018.

[56] F. Denoyelle, S. Marlin, D. Weil et al., "Clinical features of the prevalent form of childhood deafness, DFNB1, due to a connexin-26 gene defect: implications for genetic counselling," Lancet, vol. 353, no. 9161, pp. 1298-1303, 1999.

[57] X. Z. Liu, A. Pandya, S. Angeli et al., "Audiological features of GJB2 (connexin 26) deafness," Ear and Hearing, vol. 26, no. 3, pp. 361-369, 2005.

[58] K. A. King, T. Makishima, C. K. Zalewski et al., "Analysis of auditory phenotype and karyotype in 200 females with turner syndrome," Ear and Hearing, vol. 28, no. 6, pp. 831-841, 2007.

[59] N. Y. Bu-Saba, E. E. Rebeiz, S. D. Salman, A. R. Thornton, and C. West, "Significance of false-positive auditory brainstem response: a clinical study," The American Journal of Otology, vol. 15, no. 2, pp. 233-236, 1994.

[60] R. A. Hendrix, R. M. Dedio, and A. P. Sclafani, "The use of diagnostic testing in asymmetric sensorineural hearing loss," Otolaryngology-Head and Neck Surgery, vol. 103, no. 4, pp. 593-598, 2016.

[61] S. L. Urben, M. S. Benninger, and N. D. Gibbens, "Asymmetric sensorineural hearing loss in a community-based population," Otolaryngology-Head and Neck Surgery, vol. 120, no. 6, pp. 809-814, 2016.

[62] R. H. Margolis and G. L. Saly, “Toward a standard description of hearing loss," International Journal of Audiology, vol. 46, no. 12, pp. 746-758, 2009.

[63] R. H. Margolis and G. L. Saly, "Asymmetric hearing loss," Otology \& Neurotology, vol. 29, no. 4, pp. 422-431, 2008.

[64] M. G. V. C. Mazzoli, G. Van Camp, V. Newton, N. Giarbini, F. Declau, and A. Parving, "Recommendations for the description of genetic and audiological data for families with nonsyndromic hereditary hearing impairment," Audiological Medicine, vol. 1, no. 2, pp. 148-150, 2009. 